\title{
Anpassungs- und Belastungsstörungen
}

$\mathrm{D}$ ie Kategorie F43 („Reaktionen auf schwere Belastungen und Anpassungsstörungen“) der ICD-10 unterscheidet sich von allen anderen Diagnosegruppen durch das Charakteristikum einer Belastung, eines kausalen Faktors also, der Voraussetzung für diese Störungen ist. Dies erscheint auf den ersten Blick die Diagnose zu erleichtern, allerdings sind die hier zusammengefassten Erkrankungen sehr heterogen. Angesichts von Naturkatastrophen, Terroranschlägen, aber auch der $\mathrm{Zu}$ nahme der alltäglichen Gewalt vieldiskutierte Diagnosen wie die Posttraumatische Belastungsstörung finden sich hier sowie weniger spektakuläre Bilder wie die Anpassungsstörungen.

Im Jahr 2004 entschlossen wir uns aufgrund der Breite dieser Störungsgruppe, aber auch aus dem Eindruck einer diagnostischen Unschärfe und Begriffsverwirrung heraus, im Rahmen der traditionellen „Wernecker Schlossgespräche“ diese Störung näher darzustellen. Hierzu luden wir namhafte Experten ein.

Die Grundlage der Vortragsserie und des nun hier vorliegenden Schwerpunktheftes bildete Prof. Stieglitz aus Basel, der allgemeine diagnostische Regeln darlegt, hier insbesondere die Rolle der Belastungen bzw. der Traumata, auch unter dem Gesichtspunkt der Schwere. Frau Dr. Nyberg, ebenfalls aus Basel, stellt systematisch die Posttraumatische Belastungsstörung dar, insbesondere geht sie der Frage nach, inwiefern Kriseninterventionen unmittelbar nach einem Trauma sinnvoll sind. Prof. Linden aus Berlin stellt die von ihm abgegrenzte und erstbeschriebene, bisher nicht in der ICD-10 berücksichtigte, heftig diskutierte Posttraumatische Verbitterungsstörung dar. Linden geht auch auf erste epidemiologische Untersuchungen zu diesem Krankheitsbild ein, gewährt uns somit einen Blick in die von ihm geleitete empirische Forschung zu diesem Krankheitsbild. Prof. Wolfersdorf aus Bayreuth nähert sich dem Thema Anpassungsstörung von einem Diagnose-historischen Standpunkt aus, indem er fragt, was aus der „guten alten neurotischen Depression" geworden ist, inwiefern sich also dieses uns so gut vertraute Krankheitsbild in den aktuellen diagnostischen Manualen wiederfindet, und auch, welche Aspekte nun verloren gegangen sind.

Prof. Keupp, Sozialpsychologe aus München, schließlich, nähert sich dem Thema aus einer gesellschaftskritischen Perspektive: Er geht auf die vielfältigen Belastungen und Orientierungsschwierigkeiten für das Individuum ein, die sich aus gesellschaftlichen Veränderungs- und Modernisierungsprozessen ergeben. Vor diesem Hintergrund sei es ein wesentliches Qualitätsmerkmal psychiatrischen und psychotherapeutischen Arbeitens „Empowerment“ und soziale Partizipation zu fördern.

Die in diesem Heft aufgenommenen Beiträge können nicht das gesamte Spektrum der Anpassungs- und Belastungsstörungen abbilden, gehen aber auf die wichtigsten Aspekte ein. Hiermit kann ein Annäherung, und das hoffen und wünschen wir uns, an diese sehr interessante, aber mitunter vernachlässigte Störungsgruppe gelingen.

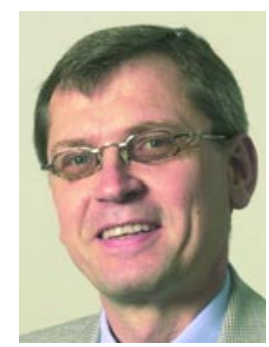

Prof. Hans-Peter Volz, Werneck

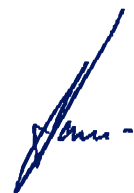

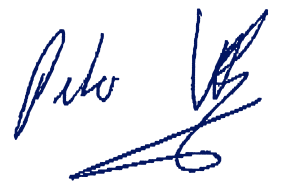

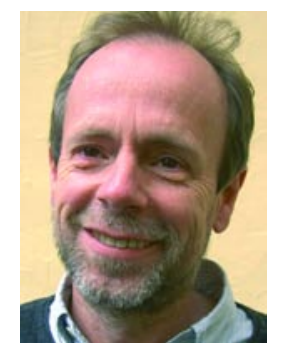

Dr. Thomas Schmelter M.A., Werneck 